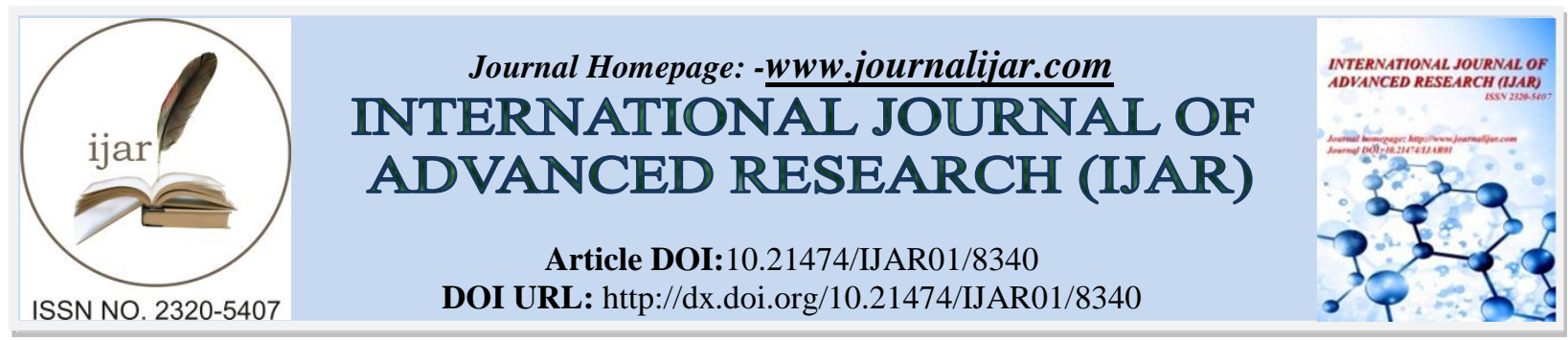

RESEARCH ARTICLE

\title{
A PROPOS DE LA GYNECOMASTIE : QUELLE PRISE EN CHARGE?
}

H.Moata, S.Rafi, G.El Mghari and N.El Ansari.

Service d'Endocrinologie Diabétologie Maladies Métaboliques et Nutrition Laboratoire PCIM, FMPM, Université Cadi Ayyad .CHU Mohamed VI. Marrakech.

\section{Manuscript Info}

Manuscript History

Received: 08 November 2018

Final Accepted: 10 December 2018

Published: January 2019

\section{Key words:-}

gynécomastie ;pseudogynécomastie ; prise en charge.

\begin{abstract}
La gynécomastie, se défini comme la prolifération bénigne du tissu glandulaire mammaire mâle, elle est généralement causée par une activité accrue des estrogènes, une diminution de l'activité de la testostérone ou l'utilisation de nombreux médicaments. Bien qu'il s'agisse d'une présentation assez courante et surtout d'étiologie bénigne, elle peut causer une anxiété considérable chez les patients. L'étape initiale consiste à exclure la pseudogynécomastie par un interrogatoire bien menu et un examen physique minutieux. Une approche par étapes qui inclut l'imagerie et les tests de laboratoire pour exclure les néoplasmes et les endocrinopathies peut faciliter le diagnostic étiologique. Si les résultats de toutes les études sont normaux, une gynécomastie idiopathique est retenue.
\end{abstract}

Copy Right, IJAR, 2018,. All rights reserved.

\section{Introduction:-}

La gynécomastie est définie comme une prolifération bénigne du tissu glandulaire mammaire masculin 1. Elle doit être distinguée de la lipomastie et du carcinome mammaire. La gynécomastie asymptomatique est très fréquente sa prévalence est de $60 \%$ à $90 \%$ chez les nouveau-nés, de $50 \%$ à $60 \%$ chez les adolescents et jusqu'à $70 \%$ chez les hommes âgés de 50 à 69 ans. La prévalence de la gynécomastie symptomatique est nettement plus faible. 5

L'évaluation diagnostique implique des tests de laboratoire ainsi que des radiographies; par conséquent, un algorithme de diagnostic qui facilite l'évaluation étape par étape peut être très rentable.

Cet article décrit la physiopathologie, les mécanismes et les causes de la gynécomastie bénigne et introduit un algorithme de diagnostic pour faciliter l'évaluation et la gestion des cas symptomatiques qui consulte.

\section{Physiopathologie}

Le déséquilibre entre l'action des œstrogènes et l'action des androgènes au niveau du tissu mammaire semble être l'étiologie principale de la gynécomastie.1 Les taux élevés d'œstrogènes sériques peuvent être le résultat de néoplasmes sécrétant des œstrogènesou de leurs précurseurs ( tumeurs productrices de gonadotrophine chorionique [hCG] et tumeurs corticosurrénaliennes) mais le plus souvent sont dus a une augmentation de la conversion des androgènes en œstrogènes par l'aromatase tissulaire (comme cela se produit dans l'obésité). Les niveaux de testostérone sérique libre diminués chez les patients présentant une insuffisance gonadique, qui peut être primaire (syndrome de Klinefelter, orchiteourlienne, castration) ou secondaire (maladie hypothalamique et hypophysaire)

Corresponding Author:-H.Moata.

Address:-Service d'Endocrinologie Diabétologie Maladies Métaboliques et Nutrition Laboratoire PCIM, FMPM, Université Cadi Ayyad . CHU Mohamed VI. Marrakech. 
ainsi que les syndromes de résistance aux androgènes dus à une altération de l'activité des enzymes impliquées dans la biosynthèse de la testostérone sont également associés à la gynécomastie.7

L'équilibre entre la testostérone libre et l'œstrogène est également affecté par les taux sériques de globuline liant les hormones sexuelles, mécanisme proposé dans la gynécomastie secondaire a l'hyperthyroïdie, les maladies hépatiques chroniques et l'utilisation de certains médicaments comme la spironolactone.6 Les récepteurs d'androgènes peuvent également présenter des anomalies génétiques ou être bloqués par certains médicaments (par exemple, le bicalutamide, utilisé dans le traitement du cancer de la prostate), et les récepteurs des œstrogènes peuvent être activés par certains médicaments ou expositions environnementales.

De ce fait, l'exposition au déséquilibre hormonal entraîne une prolifération des tissus glandulaires, c'est-à-dire une hyperplasie canalaire.

\section{Manifestations Cliniques Et Diagnostic}

L'anamnèse et l'examen physique révèlent souvent que les patients présentent en fait une pseudogynecomastie, ce qui signifie une accumulation de graisse sous-aréolaire sans prolifération réelle du tissu glandulaire. L'examen de ces patients révèle une augmentation mammaire diffuse sans nodule palpable sous-aréolaire. Ces patients n'ont pas besoin d'un traitement supplémentaire et n'ont besoin que d'être rassurés. La gynécomastie est généralement bilatérale 9 . Quand il existe une masse palpable unilatérale, dur, fixe, périphérique au mamelon associé à un écoulement, changement de la peau, ou lymphadénopathie, le cancer du sein doit être suspectée et une évaluation approfondie est recommandée. Les mesures anthropométriques ( par exemple, l'indice de masse corporelle) peuvent également être utiles parceque l'obésité peut être associée à la conversion périphérique accrue des androgènes en œstrogènes et est associée à une prévalence plus élevée de gynécomastie.3,10 La présence de varicocèle est fortement associée à la gynécomastie . Des antécédents familiaux de gynécomastie ont été observés chez 58\% des patients atteints de gynécomastie pubertaire persistante. L'interrogatoire révèle ou pas une association claire avec une consommation d'alcool et de drogues illicites ou avec un médicament et permet d'éviter une évaluation étendue et coûteuse (Tableau 1).

\begin{tabular}{|c|c|}
\hline \multirow{2}{*}{$\begin{array}{l}\text { Anti-androgènes/inhibiteurs de la synthèse des androgènes } \\
\text { - Acétate de cyprotérone } \\
\text { - Flutamide, bicalutamide } \\
\text { - Finastéride } \\
\text { - Spironolactone } \\
\text { - Kétoconazole } \\
\text { - Traitement anti-VIH (éfavirenz, antiprotéases) }\end{array}$} & $\begin{array}{l}\text { - Amphétamines } \\
\text { - Héroìne, méthadone } \\
\text { - Marijuana }\end{array}$ \\
\hline & \multirow{2}{*}{$\begin{array}{l}\text { Hormones } \\
\text { - Androgènes, œstrogènes } \\
\text { - Stéroïdes anabolisants } \\
\text { - hCG } \\
\text { - Hormone de croissance }\end{array}$} \\
\hline \multirow{2}{*}{$\begin{array}{l}\text { Antibiotiques } \\
\text { - Ethionamide } \\
\text { - Isoniazide } \\
\text { - Métronidazole }\end{array}$} & \\
\hline & \multirow{2}{*}{$\begin{array}{l}\text { Psychotropes } \\
\text { - Diazépam } \\
\text { - Halopéridol } \\
\text { - Phénothiazines } \\
\text { - Antidépresseurs tricycliques }\end{array}$} \\
\hline \multirow{2}{*}{$\begin{array}{l}\text { Antiulcéreux } \\
\text { - Cimétidine, ranitidine } \\
\text { - Oméprazole }\end{array}$} & \\
\hline & \multirow{3}{*}{$\begin{array}{l}\text { Divers } \\
\text { - IEC (énalapril, captopril) } \\
\text { - Anticalciques (nifédipine) } \\
\text { - Amiodarone, digitoxine } \\
\text { - Métoclopramide } \\
\text { - Phénytoïne } \\
\text { - Théophylline }\end{array}$} \\
\hline $\begin{array}{l}\text { Agents chimiothérapeutiques } \\
\text { - Agents alkylants } \\
\text { - Méthotrexate } \\
\text { - Vincristine, cisplatine, imatinib }\end{array}$ & \\
\hline $\begin{array}{l}\text { Toxiques } \\
\text { - Alcool }\end{array}$ & \\
\hline
\end{tabular}

Tableau 1:-médicaments et toxines causant la gynécomastie12

\section{Approche diagnostique}

Après l'histoire clinique et l'examen excluent la pseudo gynecomastie , la mammographie peut différencier entre gynécomastie vraie, une adipomastie et une masse qui nécessite un prélèvement pour exclure une tumeur maligne. La sensibilité et la spécificité de la mammographie pour les affections mammaires bénignes et malignes dépassent $90 \%$. 
Les examens de laboratoire sont poursuivis en cas de vraie gynécomastie sans explication claire. Les tests de la fonction hépatique, rénale et thyroïdienne se révèle nécessaire pour guider l'étiologie. La mesure du niveaux de la testostérone , l'estradiol, la prolactine, l'hormonelutéinisante et hCG, peut orienter vers l'hypophyse, gonade ,néoplasmes gonadiques ...

Si tous les tests sont negatives, unegynécomastie idiopathique est retenue (Tableau 2)

\begin{tabular}{|c|c|}
\hline $\begin{array}{l}\text { Gynécomasties } \\
\text { - Du nouveau-né } \\
\text { - De l'adolescent } \\
\text { - De l'homme âgé (>50 ans) }\end{array}$ & $\begin{array}{l}60-90 \% \\
50-70 \% \\
>50 \%\end{array}$ \\
\hline Gynécomasties non physiologiques & Fréquence \\
\hline Idiopathique & $25 \%$ \\
\hline Gynécomastie de la puberté persistante & $25 \%$ \\
\hline Médicaments & $25 \%$ \\
\hline Cirrhose/malnutrition & $8 \%$ \\
\hline $\begin{array}{l}\text { Hypogonadisme } \\
\text { - Primaire (infection, traumatisme, chirurgie. } \\
\text { radiothérapie, cause toxique ou génétique...) } \\
\text { - Secondaire (pathologies hypothalamo-hypophysaires, } \\
\text { hyperprolactinémie, radiothérapie, cause génétique...) }\end{array}$ & $\begin{array}{l}8 \% \\
2 \%\end{array}$ \\
\hline $\begin{array}{l}\text { Tumeurs } \\
\text { - Testiculaires (tumeurs germinales, des cellules de } \\
\text { Leydig ou des cellules de Sertoli) } \\
\text { - Surrénaliennes (carcinomes) } \\
\text { - Autres (poumons, foie...) }\end{array}$ & $\begin{array}{l}3 \% \\
\text { Rare } \\
\text { Rare }\end{array}$ \\
\hline Hyperthyroïdie & $1-2 \%$ \\
\hline Insuffisance rénale chronique & $1 \%$ \\
\hline
\end{tabular}

Tableau 2:-étiologies des gynécomasties12

\section{Diagnostic différentiel}

Le diagnostic différentiel d'une masse mammaire palpable chez un patient masculin comprend la pseudogynécomastie, la gynécomastie, le cancer du sein et de nombreuses autres affections bénignes notamment les lipomes, les kystesdermoïdes, les kystessébacés, l'inflammation lymphoplasmocytaire, l'ectasie canalaire, les hématomes et la nécrose graisseuse.12

\section{Gestion Et Pronostic}

Dans l'ensemble, la gynécomastie est une affection bénigne. Au fil du temps, le tissu fibrotique remplace la prolifération symptomatique du tissu glandulaire. Si le bilan approprié ne révèle pas de pathologie sous-jacente importante, il est recommandé de rassurer les patients et de leur assurer un suivi périodique. Bien qu'il n'yait pas de preuve d'une recommandation pour les intervalles de suivi, 6 mois semble raisonnable. Les médicaments causatifs doivent être retirés et les conditions médicales causales sous-jacentes (par exemple, hyperthyroïdie) doivent être traitées. La plupart des cas de gynécomastie pubertaire disparaissent généralement en moins d'un an $\underline{8}$. Si la gynécomastie persiste et est associée à une douleur ou à une détresse psychologique et si le patient souhaite poursuivre le traitement, des options pharmacologiques et chirurgicales sont disponibles. La pharmacothérapie est probablement bénéfique si elle est mise en œuvre tôt avant que le tissu fibreux remplace le tissu glandulaire, alors que la chirurgie peut être effectuée à tout moment. 


\section{Conclusion:-}

L'évaluation des gynécomasties peut être complexe. Une approche par étapes qui commence par une prise d'antécédents et un examen physique minutieux peut éviter le besoin d'un traitement approfondi. L'imageries élective ultérieure et les tests biologiques permettent d'exclure les néoplasmes et les endocrinopathies. L'étiologie est généralement bénigne.

\section{References:-}

1. Braunstein GD. Aromatase and gynecomastia. EndocrRelat Cancer 1999;6(2):315-324

2. Braunstein GD. Gynecomastia. N Engl J Med. 1993;328(7):490-495

3. Braunstein GD. Environmental gynecomastia [editorial]. EndocrPract. 2008;14(4):409-410

4. Ersöz H, OndeME, Terekeci H, Kurtoglu S, Tor H. Causes of gynaecomastia in young adult males and factors associated with idiopathic gynaecomastia. Int J Androl. 2002;25(5):312-316

5. Prado AC, Castillo PF. Minimal surgical access to treat gynecomastia with the use of a power-assisted arthroscopic-endoscopic cartilage shaver. PlastReconstr Surg. 2005;115(3):939-942 [

6. Braunstein GD. Gynecomastia. N Engl J Med. 2007;357(12):1229-1237

7. Hines SL, Tan WW, Yasrebi M, DePeri ER, Perez EA. The role of mammography in male patients with breast symptoms. Mayo Clin Proc. 2007;82(3):297-300

8. Alagaratnam TT. Idiopathic gynecomastia treated with tamoxifen: A preliminary report. ClinTher. 1987;9:4837.

9. Plourde PV, Reiter EO, Jou HC, Desrochers PE, Rubin SD, Bercu BB, et al. Safety and efficacy of anastrozole for the treatment of pubertal gynecomastia: A randomized, double-blind, placebo-controlled trial. J ClinEndocrinolMetab. 2004;89:4428-33.

10. Kuhn JM, Roca R, Laudat MH, Rieu M, Luton JP, Bricaire H. Dies on the treatment of idiopathic gynaecomastia with percutaneous dihydrotestosterone. ClinEndocrinol (Oxf) 1983;19:513-20

11. Eberle AJ, Sparrow JT, Keenan BS. Treatment of persistent pubertal gynecomastia with dihydrotestosteroneheptanoate. J Pediatr. 1986;109:144-9.

12. Patrick Meyer;Evaluation et prise en charge d'unegynécomastie .Rev Med Suisse 2009; volume 5.783787.2009

13. Narula HS, Carlson HE. Gynecomastia. EndocrinolMetabClin N Am 2007;36:497-519 